\title{
Genome-Wide Identification of Circular RNAs as a Novel Class of Putative Biomarkers for an Ocular Surface Disease
}

\author{
Xiu-Miao Lia Hui-Min Ge ${ }^{a, b}$ Jin Yao ${ }^{a, b}$ Yun-Fan Zhou ${ }^{a}$ Mu-Di Yao \\ Chang Liu ${ }^{b}$ Hai-Tao Hu ${ }^{b}$ Yun-Xi Zhu ${ }^{b}$ Kun Shan ${ }^{c}$ Biao Yanc Qin Jianga,b \\ ${ }^{a}$ The Affiliated Eye Hospital of Nanjing Medical University, Nanjing, ${ }^{b}$ The Fourth School of Clinical \\ Medicine, Nanjing Medical University, Nanjing, ' Eye Institute, Eye \& ENT Hospital, Shanghai Medical \\ College, Fudan University, Shanghai, China
}

\author{
Key Words \\ Pterygium • Circular RNA • Pterygium fibroblast • Pterygium epithelium
}

\begin{abstract}
Background/Aims: Pterygium is a common ocular surface disease with an unknown etiology and threatens vision as it invades into the cornea. Circular RNAs (circRNAs) are a novel class of RNA transcripts that participate in several physiological and pathological processes. However, the role of circRNAs in pathogenesis of pterygium remains largely unknown. Methods: Genome-wide circRNA expression profiling was performed to identify pterygium -related circRNAs. GO analysis, pathway analysis, and miRNA response elements analysis was performed to predict the function of differentially expressed circRNAs in pterygium. MTT assays, Ki67 staining, Transwell assay, Hoechst 33342 staining, and Calcein-AM/PI staining were performed to determine the effect of circRNA silencing on pterygium fibroblast and epithelial cell function. Results: Approximately 669 circRNAs were identified to be abnormally expressed in pterygium tissues. GO analysis demonstrated that the host genes of differentially expressed circRNAs were targeted to extracellular matrix organization (ontology: biological process), cytoplasm (ontology: cellular component), and protein binding (ontology: molecular function). Pathway analysis showed that dysregulated circRNAs-mediated regulatory networks were mostly enriched in focal adhesion signaling pathway. Notably, circ_0085020 (circLAPTM4B) was shown as a potential biomarker for pterygium. circ_0085020 (circ-LAPTM4B) silencing affected the viability, proliferation, migration, and apoptosis of pterygium fibroblast and epithelial cells in vitro. Conclusions: This study provides evidence that circRNAs are involved in the pathogenesis of pterygium and might constitute promising targets for the therapeutic intervention of pterygium.

Xiu-Miao Li, Hui-Min Ge and Jin Yao contributed equally to this work.

\section{KARGER}




\section{Cellular Physiology Cell Physiol Biochem 2018;47:1630-1642 \begin{tabular}{l|l} 
and Biochemistry 10.1159/000490982 & $\begin{array}{l}\text { O 2018 The Author(s). Published by S. Karger AG, Basel } \\
\text { www.karger.com/cpb }\end{array}$
\end{tabular} \\ Li et al.: CircRNA and Pterygium}

\section{Introduction}

Pterygium is a common ocular surface disease only observed in humans. Pterygium is characterized by the overgrowth of conjunctiva into the cornea. It affects visual function in the advanced cases, leading to redness and irritation [1]. Although the pathogenesis of pterygium is still uncertain, recent studies have provided some insights into the pathogenesis. Aberrant cell proliferation, defects in wound healing, cell transformation, abnormal matrix remodeling, angiogenesis, oxidative stress, and genetic susceptibility has been implicated in the formation and development of pterygium [2].

Current methods for pterygium treatment mainly include surgical resection combined with conjunctival autograft, mitomycin $\mathrm{C}$ administration, or other adjunctive forms of treatment [3-5]. However, the high recurrence of surgical management means that that nonsurgical method for pterygia treatment might be beneficial, particularly in these countries where medical resources are not well developed. Thus, further studies are still required to clarify the mechanism of the formation and development of pterygium.

More importantly, pterygium research has important and widespread clinical significance. The pathogenesis of pterygium is usually associated with abnormal fibroblast proliferation and matrix deposition. Its clinical severity is determined by the amount of fibrous tissues, implying that pterygium is a type of fibrotic disease [6, 7]. Epidemiological data, ray tracing models and histopathological changes have shown that chronic UV exposure is a widely accepted aetiological factor in the pathogenesis of pterygium. Pterygium shares many common features with UV damaged skin. Compared to other organs affected by fibrosis or photo-damage, it is accessible to obtain human pterygium tissues through surgical excision $[8,9]$. Thus, pterygium is also a very suitable and relevant model for dissecting the molecular mechanism of fibrosis and photo-damage. Clarifying the mechanism of pterygium formation would also have important implications for the pathogenesis of other diseases, such as skin photo-damage, liver, kidney, and lung fibrotic diseases.

Circular RNAs (circRNAs) is a class of non-coding RNAs that are widely expressed in mammals. They are generated by the joining of the 5' splice site with the 3' splice site via back-splicing [10]. circRNA expression is often highly conserved across species and appear highly stable comparing to their linear parental transcripts $[11,12]$. Circular RNAs participate in a wide range of developmental and physiological processes, including fibrosis, cell proliferation, apoptosis, differentiation, and angiogenesis [13, 14]. Abnormal circRNA expression has been shown in several human disorders, such as cancers, inflammatory diseases, and neurological diseases [15-17]. However, the characterization and function of circRNAs in pterygium is still unknown.

In this study, we collected the paired pterygium and normal conjunctiva to determine the role of circRNAs in the pathogenesis of pterygium. Approximately 669 circRNAs were found to be abnormally expressed in pterygium tissues. We characterized one circular, circ_0085020 (circ-LAPTM4B), which was significantly up-regulated in pterygium tissues. Functional assays revealed that circ_0085020 (circ-LAPTM4B) could regulate pterygium fibroblast and epithelial cell function in vitro. This study shows that circRNAs are potential targets for the therapeutic intervention of pterygium.

\section{Materials and Methods}

\section{Specimen collection}

This study was approved by Nanjing Medical University Committee for Research Ethics. All patients were given the informed consent before inclusion. The inclusion criteria were the presence of untreated primary pterygium, clinical indication for conventional excision of pterygium with auto-transplantation of conjunctiva. The corneal ingrowth in patients was ranging from $2 \mathrm{~mm}$ to $4 \mathrm{~mm}$. A small piece of normal conjunctival tissue was excised as control tissue. The collected samples were transferred to $1.5 \mathrm{ml}$ tubes and stored at $-80{ }^{\circ} \mathrm{C}$ for further analysis. 


\section{Cellular Physiology Cell Physiol Biochem 2018:47:1630-1642 \begin{tabular}{l|l|l} 
DOI: 10.1159/000490982 & $\begin{array}{l}\text { () 2018 The Author(s). Published by S. Karger AG, Basel } \\
\text { www.karger.com/cpb }\end{array}$ \\
\hline
\end{tabular} \\ Li et al.: CircRNA and Pterygium}

RNA isolation

Total RNA was isolated from the clinical samples using TRIzol reagent (Ambion, Austin, TX, US) according to the manufacturer's protocol. The integration of RNAs for microarray analysis was estimated by the Agilent Bioanalyzer 2100 (Agilent technologies, Santa Clara, CA, US). The purity and quantity of other RNAs was estimated by the NanoDrop ND-1000 (NanoDrop, Wilmington, USA). The integrity of total RNAs was also estimated by the electrophoresis on denaturing agarose gel.

\section{Microarray analysis}

Total RNAs were extracted from 3 paired pterygium and normal conjunctiva. The corneal ingrowth in patients was $4 \mathrm{~mm}, 3 \mathrm{~mm}$, and $4.5 \mathrm{~mm}$, respectively. The patients include two males (age: 48 and 54) and one female (age: 46). Total RNAs were treated with RNase R to remove the linear RNAs. The enriched circRNAs were amplified and transcribed into fluorescent cRNA, and then purified using the RNeasy Mini Kit (Qiagen, Hilden, Germany). The Cy3-labeled cRNAs were hybridized onto the SBS Human Circular RNA Microarray using Gene Expression Hybridization Kit (Agilent technologies, Santa Clara, CA, US). The slides were washed after hybridization, and scanned by Agilent Microarray Scanner (Agilent technologies, Santa Clara, CA, US). All data were extracted using the Feature Extraction software 10.7 (Agilent technologies, Santa Clara, CA, US).

\section{Bioinformatics analysis}

Circular RNA expression profiles were compared by two-way ANOVA. The expression level was given as average normalized (log transformed) signal intensity. Correction for multiple testing was conducted using the false discovery rate (FDR) controlling procedure. GO analysis and pathway analysis of the host genes of circRNAs was performed using the Database for Annotation, Visualization, and Integrated Discovery (DAVID; http://david.abcc.ncifcrf.gov). The binding pattern between miRNAs and circRNAs were predicted based on the miRanda and TargetScan algorithm.

\section{Quantitative real-time reverse transcription polymerase chain reaction ( $q R T-P C R$ )}

Total RNAs from the pterygium and normal conjunctival tissues were reversely transcribed using PrimeScript RT reagent Kit (Takara). qPCRs were conducted using SYBR Premix Ex Taq (Takara) in the PikoReal Real-Time PCR System (Thermo Scientific). The divergent primers for circRNA quantification were designed based on the backsplice junction sequence of circRNAs. The relative expression change of each circRNA was calculated by the cycle threshold method ( $\Delta \Delta \mathrm{Ct}$ method).

\section{Isolation and culture of pterygium epithelium and fibroblast}

Primary epithelial and fibroblasts were isolated using the explants methods with slight modifications $[18,19]$. In brief, the fresh pterygium sample was cut into small pieces (1-2 $\mathrm{mm}$ in diameter), washed in Hank's solution, and placed in a culture dish as explants in DMEM/F12 medium supplemented with 10\% FBS, $100 \mathrm{U} / \mathrm{mL}$ penicillin, and $100 \mathrm{mg} / \mathrm{mL}$ streptomycin. Epithelial cells migrated from explants between 3 and 5 days and showed cobblestone morphology. Fibroblasts migrated from the pterygium body between 7 to 14 days and showed elongated spindle-shaped morphology.

\section{Cell viability assay}

Cell viability was determined using 3-(4, 5-dimethylthiazol-2-yl)-2, 5- diphenyl-tetrazolium-bromide assay (MTT) [20]. Briefly, pterygium epithelium or fibroblasts were plated at a density of about $1.5 \times 10^{4}$ cells per well in 96-well plates. After the required treatments, these cells were incubated with MTT (0.5 mg/ $\mathrm{ml}$ ) for $3 \mathrm{~h}$ at $37^{\circ} \mathrm{C}$, and then DMSO solution was added to dissolve formazan crystals. The absorbance was detected at absorbance of $570 \mathrm{~nm}$ by a microplate reader (Molecular Devices).

\section{Hoechst staining}

After the required treatments, pterygium epithelium or fibroblast were fixed in $4 \%$ formaldehyde for $10 \mathrm{~min}$, and then permeabilized using Triton-X 100 for $15 \mathrm{~min}$. These cells were stained with Hoechst $33342(150 \mu \mathrm{g} / \mathrm{ml})$ for $10 \mathrm{~min}$, and then washed with PBS buffer. The staining result was observed using an Olympus IX-73 microscope [21]. 


\section{Cellular Physiology Cell Physiol Biochem 2018;47:1630-1642

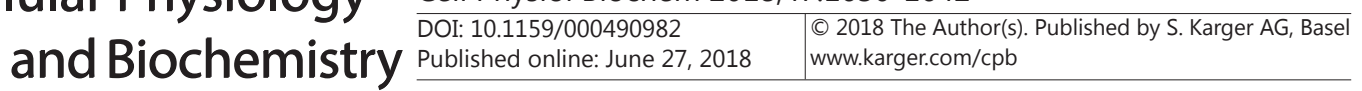 Li et al.: CircRNA and Pterygium}

Propidium iodide (PI)/Calcein-AM double staining

After the required treatments, pterygium epithelium or fibroblasts were fixed with $4 \%$ formaldehyde for $10 \mathrm{~min}$, stained with Calcein-AM solution $(10 \mu \mathrm{mol} / \mathrm{L})$ for $10 \mathrm{~min}$, and then stained with PI solution $(10$ $\mu \mathrm{mol} / \mathrm{L}$ ) for additional $10 \mathrm{~min}$. The live cells were observed at the excitation filter of $490 \mathrm{~nm}$. Apoptotic cells were observed at the excitation filter of $545 \mathrm{~nm}[22]$.

\section{Cell migration assay}

Pterygium epithelium or fibroblasts were placed in the upper chamber of a Transwell $\left(5 \times 10^{4}\right.$ cells per chamber, Corning) coated with fibronectin $(1 \mu \mathrm{g} / \mathrm{ml})$. After the required treatment, pterygium epithelium or fibroblasts were allowed for migrating for $8 \mathrm{~h}$ at $37^{\circ} \mathrm{C}$. The non-migrated cells were scraped off the upper surface of the membrane. The migrated cells remaining on the bottom surface were stained with the crystal violet, and then observed using an Olympus IX-73 microscope [23].

\section{Statistical analysis}

The statistical difference was determined by Student's $t$ test (when 2 groups were compared) or oneway ANOVA test (when $>2$ groups were compared). Post hoc Tukey analyses were performed when $P<0.05$. $P<0.05$ was considered statistically significant.

\section{Results}

Identification of differentially expressed circRNAs between normal conjunctiva and pterygium

To identify pterygium-related circular RNAs, we performed circRNA expression profiles between normal conjunctiva and pterygium tissues using the SBC Human $(4 \times 180 \mathrm{~K})$ Circular RNA Microarray (Shanghai Biotechnology Co., Ltd.). The box plots show the distribution of expression levels for all samples in the circRNA microarray dataset (Fig. 1A). We used the volcano plot to filter differentially expressed circRNAs between normal conjunctiva and pterygium tissues (Fig. 1B). We set a threshold as fold change greater than 2.0, and identified 669 differentially expressed circRNAs, including 341 up-regulated circRNAs and 328 down-regulated circRNAs (Table 1, conjunctiva versus pterygium). We then used the Top 20 dysregulated circRNAs to perform hierarchical clustering analysis. The up-regulated or down-regulated circRNAs were clustered into the same samples (Fig. 1C). To verify the results of microarray data, we randomly selected 20 dysregulated

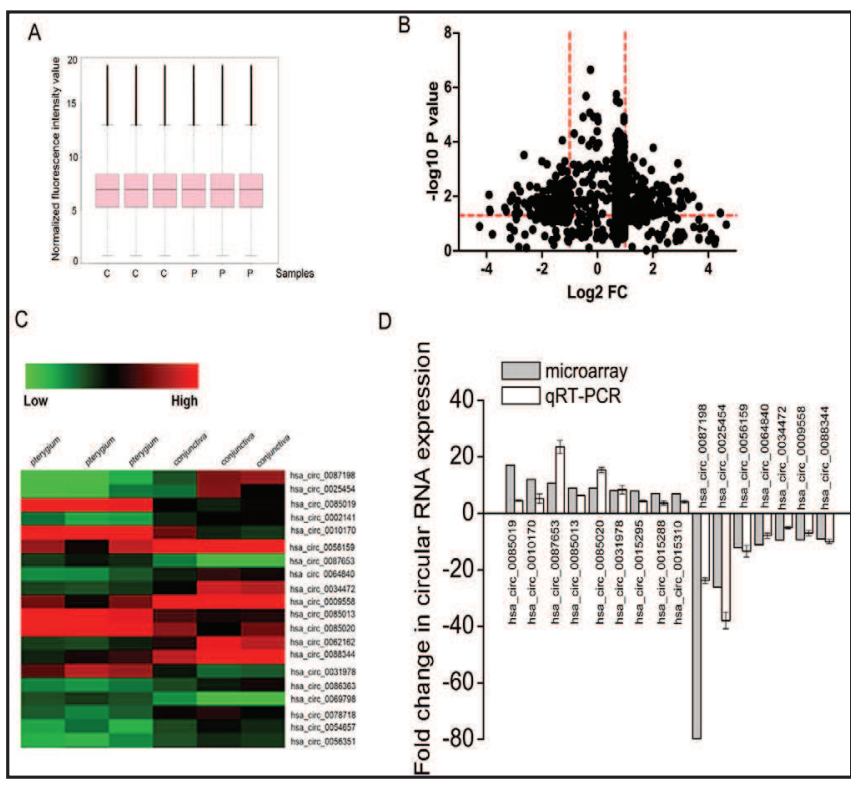

Fig. 1. Identification of differentially expressed circRNAs between normal conjunctiva and pterygium (A) The box plot shows the distribution of circular RNA expression levels for all samples in the microarray dataset. The box plot consists of boxes with a central line and two tails. The central line represents the median of the data, whereas the tail represents the upper and lower quartiles. (B) The volcano plot shows differentially expressed circRNAs between normal conjunctiva and pterygium samples. (C) Heatmaps were generated through the hierarchical cluster analysis to show the top 20 dysregulated circRNAs between normal conjunctiva and pterygium samples. Red indicates up-regulated circular RNAs, and green indicates down-regulated circular RNAs. (D) qRT-PCRs were used to detect the expression pattern of the top 20 dysregulated circRNAs using the normal conjunctiva samples and pterygium samples for microarray. 


\begin{tabular}{|c|c|c|}
\hline yy & Cell Physiol Biochem 2018; & 17:1630-1642 \\
\hline and Biochemistry & $\begin{array}{l}\text { DOI: } 10.1159 / 000490982 \\
\text { Published online: June 27, } 2018\end{array}$ & $\begin{array}{l}\text { O } 2018 \text { The Author(s). Published by S. Karger AG, Basel } \\
\text { www.karger.com/cpb }\end{array}$ \\
\hline
\end{tabular}

Li et al.: CircRNA and Pterygium

Table 1. Differentially expressed circRNAs between paired conjunctiva and pterygium

\begin{tabular}{|c|c|c|c|c|c|c|c|}
\hline $\begin{array}{r}\text { cicRNA } \\
\text {. }\end{array}$ & Fold change & cicRNA & Fold change & cicRNA & Fold change & cicRNA & Fold change \\
\hline Up-regulated & & & & & & & \\
\hline hsa_circ_0085019 & 17.01 & hsa_circ_0014715 & 3.22 & hsa_circ_0060059 & 2.57 & hsa_circ_0016486 & 2.30 \\
\hline hsa_circ_0010170 & 12.01 & hsa_circ_0091055 & 3.19 & hsa_circ_0069843 & 2.57 & hsa_circ_0042594 & 2.30 \\
\hline hsa_circ_0087653 & 10.71 & hsa_circ_0035918 & 3.19 & hsa_circ_0045762 & 2.56 & hsa_circ_0014613 & 2.29 \\
\hline hsa_circ_0085013 & 8.90 & hsa_circ_0042563 & 3.18 & hsa_circ_0031778 & 2.56 & hsa_circ_0023763 & 2.29 \\
\hline hsa_circ_0085020 & 8.88 & hsa_circ_0082590 & 3.18 & hsa_circ_0031031 & 2.55 & hsa_circ_0043325 & 2.29 \\
\hline hsa_circ_0031978 & 8.05 & hsa_circ_0060231 & 3.17 & hsa_circ_0070498 & 2.55 & hsa_circ_0001145 & 2.28 \\
\hline hsa_circ_0069798 & 7.98 & hsa_circ_0015173 & 3.17 & hsa_circ_0031013 & 2.54 & hsa_circ_0081998 & 2.28 \\
\hline hsa_circ_0015295 & 7.05 & hsa_circ_0076144 & 3.12 & hsa_circ_0033397 & 2.54 & hsa_circ_0058178 & 2.28 \\
\hline hsa_circ_0015288 & 7.03 & hsa_circ_0082378 & 3.11 & hsa_circ_0059979 & 2.54 & hsa_circ_0057143 & 2.27 \\
\hline hsa_circ_0015310 & 6.90 & hsa_circ_0022450 & 3.10 & hsa_circ_0031265 & 2.54 & hsa_circ_0010098 & 2.27 \\
\hline hsa_circ_0071473 & 6.27 & hsa_circ_0007473 & 3.09 & hsa_circ_0052770 & 2.54 & hsa_circ_0063171 & 2.26 \\
\hline hsa_circ_0027279 & 6.19 & hsa_circ_0001448 & 3.08 & hsa_circ_0052775 & 2.53 & hsa_circ_0042404 & 2.26 \\
\hline hsa_circ_0088312 & 6.19 & hsa_circ_0083345 & 3.07 & hsa_circ_0019062 & 2.53 & hsa_circ_0015001 & 2.25 \\
\hline hsa_circ_0015306 & 6.09 & hsa_circ_0059911 & 3.07 & hsa_circ_0030778 & 2.52 & hsa_circ_0030629 & 2.25 \\
\hline hsa_circ_0084390 & 5.97 & hsa_circ_0036939 & 3.05 & hsa_circ_0002679 & 2.52 & hsa_circ_0042699 & 2.25 \\
\hline hsa_circ_0015305 & 5.88 & hsa_circ_0059987 & 3.05 & hsa_circ_0061373 & 2.52 & hsa_circ_0083287 & 2.25 \\
\hline hsa_circ_0008786 & 5.60 & hsa_circ_0046913 & 3.05 & hsa_circ_0030666 & 2.51 & hsa_circ_0052604 & 2.25 \\
\hline hsa_circ_0039233 & 5.48 & hsa_circ_0015860 & 3.04 & hsa_circ_0084446 & 2.51 & hsa_circ_0052570 & 2.24 \\
\hline hsa_circ_0030352 & 5.46 & hsa_circ_0009755 & 3.04 & hsa_circ_0074493 & 2.51 & hsa_circ_0052741 & 2.23 \\
\hline hsa_circ_0041603 & 5.45 & hsa_circ_0042252 & 3.03 & hsa_circ_0061372 & 2.50 & hsa_circ_0023688 & 2.23 \\
\hline hsa_circ_0056937 & 5.42 & hsa_circ_0017303 & 3.03 & hsa_circ_0054135 & 2.49 & hsa_circ_0083799 & 2.23 \\
\hline hsa_circ_0000142 & 5.17 & hsa_circ_0008019 & 2.99 & hsa_circ_0059505 & 2.48 & hsa_circ_0083560 & 2.22 \\
\hline hsa_circ_0076138 & 5.11 & hsa_circ_0070608 & 2.98 & hsa_circ_0003538 & 2.48 & hsa_circ_0057471 & 2.22 \\
\hline hsa_circ_0029427 & 5.08 & hsa_circ_0036384 & 2.95 & hsa_circ_0012617 & 2.48 & hsa_circ_0052878 & 2.21 \\
\hline hsa_circ_0087883 & 4.60 & hsa_circ_0014717 & 2.95 & hsa_circ_0072097 & 2.47 & hsa_circ_0035994 & 2.21 \\
\hline hsa_circ_0068028 & 4.55 & hsa_circ_0060636 & 2.92 & hsa_circ_0060275 & 2.47 & hsa_circ_0076987 & 2.21 \\
\hline hsa_circ_0021414 & 4.51 & hsa_circ_0008988 & 2.90 & hsa_circ_0009620 & 2.47 & hsa_circ_0088711 & 2.21 \\
\hline hsa_circ_0056875 & 4.47 & hsa_circ_0022437 & 2.90 & hsa_circ_0021902 & 2.46 & hsa_circ_0078241 & 2.20 \\
\hline hsa_circ_0004930 & 4.44 & hsa_circ_0057730 & 2.90 & hsa_circ_0068787 & 2.45 & hsa_circ_0010413 & 2.20 \\
\hline hsa_circ_0016479 & 4.37 & hsa_circ_0024717 & 2.89 & hsa_circ_0061201 & 2.45 & hsa_circ_0030036 & 2.20 \\
\hline hsa_circ_0056156 & 4.33 & hsa_circ_0017800 & 2.89 & hsa_circ_0032891 & 2.44 & hsa_circ_0047418 & 2.20 \\
\hline hsa_circ_0059375 & 4.33 & hsa_circ_0002014 & 2.89 & hsa_circ_0056614 & 2.44 & hsa_circ_0058420 & 2.20 \\
\hline hsa_circ_0087880 & 4.31 & hsa_circ_0070268 & 2.89 & hsa_circ_0061881 & 2.43 & hsa_circ_0063056 & 2.20 \\
\hline hsa_circ_0057237 & 4.27 & hsa_circ_0015435 & 2.88 & hsa_circ_0031856 & 2.43 & hsa_circ_0009700 & 2.19 \\
\hline hsa_circ_0031698 & 4.20 & hsa_circ_0008807 & 2.86 & hsa_circ_0013908 & 2.43 & hsa_circ_0028884 & 2.19 \\
\hline hsa_circ_0063794 & 4.17 & hsa_circ_0006336 & 2.85 & hsa_circ_0029423 & 2.43 & hsa_circ_0024788 & 2.19 \\
\hline hsa_circ_0087881 & 4.17 & hsa_circ_0046147 & 2.85 & hsa_circ_0021564 & 2.42 & hsa_circ_0032776 & 2.19 \\
\hline hsa_circ_0016062 & 4.11 & hsa_circ_0021916 & 2.84 & hsa_circ_0011218 & 2.42 & hsa_circ_0046932 & 2.19 \\
\hline hsa_circ_0002999 & 4.10 & hsa_circ_0070024 & 2.83 & hsa_circ_0045602 & 2.41 & hsa_circ_0034955 & 2.19 \\
\hline hsa_circ_0047466 & 4.09 & hsa_circ_0029618 & 2.83 & hsa_circ_0024180 & 2.41 & hsa_circ_0084274 & 2.18 \\
\hline hsa_circ_0014540 & 4.08 & hsa_circ_0008539 & 2.82 & hsa_circ_0059985 & 2.40 & hsa_circ_0054278 & 2.18 \\
\hline hsa_circ_0029908 & 4.05 & hsa_circ_0017285 & 2.82 & hsa_circ_0014260 & 2.40 & hsa_circ_0035916 & 2.17 \\
\hline hsa_circ_0083394 & 4.00 & hsa_circ_0080270 & 2.82 & hsa_circ_0013512 & 2.39 & hsa_circ_0083810 & 2.17 \\
\hline hsa_circ_0076145 & 3.95 & hsa_circ_0085501 & 2.82 & hsa_circ_0010811 & 2.38 & hsa_circ_0033384 & 2.16 \\
\hline hsa_circ_0036594 & 3.95 & hsa_circ_0014135 & 2.81 & hsa_circ_0052767 & 2.38 & hsa_circ_0016435 & 2.16 \\
\hline hsa_circ_0063421 & 3.86 & hsa_circ_0020299 & 2.81 & hsa_circ_0059655 & 2.38 & hsa_circ_0074887 & 2.16 \\
\hline hsa_circ_0059373 & 3.83 & hsa_circ_0056606 & 2.81 & hsa_circ_0063262 & 2.38 & hsa_circ_0043861 & 2.16 \\
\hline hsa_circ_0084770 & 3.79 & hsa_circ_0022436 & 2.81 & hsa_circ_0076995 & 2.38 & hsa_circ_0083317 & 2.15 \\
\hline hsa_circ_0065564 & 3.76 & hsa_circ_0005565 & 2.80 & hsa_circ_0054668 & 2.38 & hsa_circ_0072621 & 2.15 \\
\hline hsa_circ_0016118 & 3.70 & hsa_circ_0072256 & 2.80 & hsa_circ_0013157 & 2.37 & hsa_circ_0025852 & 2.15 \\
\hline hsa_circ_0000410 & 3.66 & hsa_circ_0022446 & 2.79 & hsa_circ_0091220 & 2.37 & hsa_circ_0056417 & 2.15 \\
\hline hsa_circ_0016187 & 3.66 & hsa_circ_0014723 & 2.77 & hsa_circ_0053883 & 2.36 & hsa_circ_0025062 & 2.15 \\
\hline hsa_circ_0021565 & 3.62 & hsa_circ_0012611 & 2.77 & hsa_circ_0000466 & 2.36 & hsa_circ_0084042 & 2.15 \\
\hline hsa_circ_0077876 & 3.60 & hsa_circ_0054903 & 2.76 & hsa_circ_0036495 & 2.35 & hsa_circ_0054753 & 2.14 \\
\hline hsa_circ_0044683 & 3.58 & hsa_circ_0051045 & 2.76 & hsa_circ_0013510 & 2.35 & hsa_circ_0001646 & 2.14 \\
\hline hsa_circ_0035980 & 3.56 & hsa_circ_0057628 & 2.74 & hsa_circ_0008763 & 2.35 & hsa_circ_0050220 & 2.13 \\
\hline hsa_circ_0082595 & 3.54 & hsa_circ_0014277 & 2.72 & hsa_circ_0034578 & 2.35 & hsa_circ_0002107 & 2.13 \\
\hline hsa_circ_0061884 & 3.52 & hsa_circ_0015141 & 2.72 & hsa_circ_0008758 & 2.34 & hsa_circ_0026138 & 2.13 \\
\hline hsa_circ_0035012 & 3.51 & hsa_circ_0013906 & 2.71 & hsa_circ_0047419 & 2.34 & hsa_circ_0031101 & 2.13 \\
\hline hsa_circ_0057923 & 3.48 & hsa_circ_0086754 & 2.71 & hsa_circ_0041929 & 2.34 & hsa_circ_0084425 & 2.12 \\
\hline hsa_circ_0035991 & 3.47 & hsa_circ_0011975 & 2.69 & hsa_circ_0026663 & 2.33 & hsa_circ_0004168 & 2.12 \\
\hline hsa_circ_0059908 & 3.46 & hsa_circ_0090981 & 2.69 & hsa_circ_0060254 & 2.33 & hsa_circ_0063590 & 2.12 \\
\hline hsa_circ_0070617 & 3.43 & hsa_circ_0014134 & 2.68 & hsa_circ_0060095 & 2.33 & hsa_circ_0078502 & 2.12 \\
\hline hsa_circ_0014270 & 3.42 & hsa_circ_0054277 & 2.66 & hsa_circ_0083290 & 2.33 & hsa_circ_0011408 & 2.12 \\
\hline hsa_circ_0039483 & 3.41 & hsa_circ_0013583 & 2.66 & hsa_circ_0021897 & 2.33 & hsa_circ_0028019 & 2.12 \\
\hline hsa_circ_0029718 & 3.33 & hsa_circ_0061678 & 2.65 & hsa_circ_0031428 & 2.33 & hsa_circ_0053274 & 2.12 \\
\hline $\begin{array}{l}\text { hsa_circ_0003959 } \\
\text { hsa }\end{array}$ & $\begin{array}{l}3.35 \\
3.33\end{array}$ & hsa_circ_0033385 & 2.65 & hsa_circ_0008836 & 2.32 & hsa_circ_0012799 & 2.10 \\
\hline hsa_circ_0089340 & 3.32 & hsa_circ_0005957 & 2.64 & hsa_circ_0059664 & 2.32 & hsa_circ_0085324 & 2.10 \\
\hline hsa_circ_0008595 & 3.32 & hsa_circ_0077869 & 2.63 & hsa_circ_0083804 & 2.32 & hsa_circ_0052029 & 2.10 \\
\hline hsa_circ_0002604 & 3.31 & hsa_circ_0062773 & 2.63 & hsa_circ_0084109 & 2.32 & hsa_circ_0078399 & 2.10 \\
\hline hsa_circ_0057728 & 3.30 & hsa_circ_0019541 & 2.62 & hsa_circ_0052713 & 2.32 & hsa_circ_0084451 & 2.10 \\
\hline hsa_circ_0023585 & 3.29 & hsa_circ_0000535 & 2.62 & hsa_circ_0025214 & 2.32 & hsa_circ_0069750 & 2.09 \\
\hline hsa_circ_0023762 & 3.27 & hsa_circ_0020360 & 2.61 & hsa_circ_0084936 & 2.31 & hsa_circ_0020705 & 2.09 \\
\hline hsa_circ_0034691 & 3.27 & hsa_circ_0022664 & 2.60 & hsa_circ_0056410 & 2.31 & hsa_circ_0059158 & 2.09 \\
\hline hsa_circ_0000101 & 3.27 & hsa_circ_0013910 & 2.60 & hsa_circ_0083288 & 2.31 & hsa_circ_0077381 & 2.08 \\
\hline hsa_circ_0017288 & 3.25 & hsa_circ_0072592 & 2.59 & hsa_circ_0079489 & 2.31 & hsa_circ_0008820 & 2.08 \\
\hline hsa_circ_0067780 & 3.24 & hsa_circ_0072265 & 2.58 & hsa_circ_0077728 & 2.30 & hsa_circ_0039258 & 2.07 \\
\hline hsa_circ_0000412 & 3.24 & hsa_circ_0013911 & 2.58 & hsa_circ_0026128 & 2.30 & hsa_circ_0064110 & 2.06 \\
\hline hsa_circ_0029711 & 3.23 & hsa_circ_0060053 & 2.58 & hsa_circ_0034758 & 2.30 & hsa_circ_0031429 & 2.06 \\
\hline hsa circ 0050051 & 3.23 & hsa circ 0076990 & 2.57 & hsa circ 0029623 & 2.30 & hsa circ 0036063 & 2.06 \\
\hline hsa_circ_0063626 & 2.05 & hsa_circ_0006979 & 2.02 & hsa_circ_0052727 & 2.05 & hsa_circ_0016799 & 2.06 \\
\hline hsa_circ_0070908 & 2.04 & hsa_circ_0027524 & 2.01 & hsa_circ_0078266 & 2.05 & hsa_circ_0022908 & 2.06 \\
\hline hsa_circ_0 084444 & 2.03 & hsa_circ_0023708 & 2.01 & hsa_circ_0043192 & 2.05 & hsa_circ_0081427 & 2.06 \\
\hline hsa_circ_0022933 & 2.03 & hsa_circ_0072788 & 2.01 & hsa_circ_0087052 & 2.05 & hsa_circ_0069496 & 2.00 \\
\hline $\begin{array}{l}\text { hsa_circ_0023518 } \\
\text { hsare }\end{array}$ & 2.03 & hsa_circ_0043211 & 2.00 & hsa_circ_0030686 & 2.00 & hsa_circ_0030167 & 2.00 \\
\hline hsa_circ_0 049078 & 2.00 & & & & & & \\
\hline
\end{tabular}

continued

circRNAs, including 10 up-regulated circRNAs and 10 down-regulated circRNAs for expression verification. 16 of 20 circRNA expression pattern were verified by qRT-PCRs, which showed similar expression pattern as previously detected by microarrays (Fig. 1D).

GO analysis and pathway analysis of the host genes of dysregulated circRNAs

The majority of circRNAs are generated from the exonic or intronic sequences of their host genes. Several circRNAs have shown as important regulators of the expression of their 


\section{Cellular Physiology Cell Physiol Biochem 2018:47:1630-1642 \begin{tabular}{ll|l} 
and Biochemistry & Dublished online: June 27, 2018 & $\begin{array}{l}\text { @ 2018 The Author(s). Published by S. Karger AG, Basel } \\
\text { www.karger.com/cpb }\end{array}$ \\
\hline
\end{tabular}}

Li et al.: CircRNA and Pterygium

\begin{tabular}{|c|c|c|c|c|c|c|c|}
\hline cicRNA & Fold change & cicRNA & Fold change & cicRNA & Fold change & cicRNA & Fold change \\
\hline \multicolumn{8}{|l|}{ Down-regulated } \\
\hline hsa_circ_0087198 & 79.74 & hsa_circ_0058201 & 2.54 & hsa_circ_0023867 & 3.52 & hsa_circ_0021876 & 2.12 \\
\hline hsa_circ_0025454 & 26.11 & hsa_circ_0045686 & 2.54 & hsa_circ_0089548 & 3.49 & hsa_circ_0005937 & 2.11 \\
\hline hsa_circ_0002141 & 12.13 & hsa_circ_0048089 & 2.54 & hsa_circ_0067022 & 3.49 & hsa_circ_0008331 & 2.11 \\
\hline hsa_circ_0056159 & 11.10 & hsa_circ_0038727 & 2.53 & hsa_circ_0023276 & 3.48 & hsa_circ_0089246 & 2.11 \\
\hline hsa_circ_0064840 & 9.40 & hsa_circ_0028128 & 2.52 & hsa_circ_0058138 & 3.46 & hsa_circ_0026337 & 2.10 \\
\hline hsa_circ_0034472 & 9.38 & hsa_circ_0035165 & 2.52 & hsa_circ_0075368 & 3.43 & hsa_circ_0021409 & 2.10 \\
\hline hsa_circ_0009558 & 9.08 & hsa_circ_0043819 & 2.52 & hsa_circ_0082048 & 3.41 & hsa_circ_0021300 & 2.10 \\
\hline hsa_circ_0062162 & 8.87 & hsa_circ_0001555 & 2.51 & hsa_circ_0058153 & 3.41 & hsa_circ_0022618 & 2.09 \\
\hline hsa_circ_0088344 & 8.20 & hsa_circ_0029345 & 2.50 & hsa_circ_0005712 & 3.39 & hsa_circ_0010591 & 2.08 \\
\hline hsa_circ_0086363 & 7.98 & hsa_circ_0065742 & 2.50 & hsa_circ_0074637 & 3.38 & hsa_circ_0021283 & 2.08 \\
\hline hsa_circ_0078718 & 7.77 & hsa_circ_0087408 & 2.50 & hsa_circ_0085788 & 3.33 & hsa_circ_0017452 & 2.08 \\
\hline hsa_circ_0054657 & 7.70 & hsa_circ_0066378 & 2.49 & hsa_circ_0030864 & 3.32 & hsa_circ_0049396 & 2.08 \\
\hline hsa_circ_0056351 & 7.60 & hsa_circ_0074324 & 2.47 & hsa_circ_0035614 & 3.29 & hsa_circ_0072068 & 2.08 \\
\hline hsa_circ_0054662 & 7.58 & hsa_circ_0029338 & 2.47 & hsa_circ_0054437 & 3.28 & hsa_circ_0021866 & 2.08 \\
\hline hsa_circ_0079555 & 7.39 & hsa_circ_0089694 & 2.47 & hsa_circ_0059361 & 3.26 & hsa_circ_0017491 & 2.08 \\
\hline hsa_circ_0090172 & 6.98 & hsa_circ_0035522 & 2.47 & hsa_circ_0025722 & 3.24 & hsa_circ_0067057 & 2.08 \\
\hline hsa_circ_0006200 & 6.89 & hsa_circ_0034277 & 2.47 & hsa_circ_0069259 & 3.23 & hsa_circ_0054324 & 2.08 \\
\hline hsa_circ_0084805 & 6.69 & hsa_circ_0057402 & 2.47 & hsa_circ_0049386 & 3.21 & hsa_circ_0021084 & 2.08 \\
\hline hsa_circ_0079554 & 6.36 & hsa_circ_0064831 & 2.46 & hsa_circ_0081217 & 3.20 & hsa_circ_0051925 & 2.08 \\
\hline hsa_circ_0002240 & 6.32 & hsa_circ_0063935 & 2.46 & hsa_circ_0001964 & 3.19 & hsa_circ_0029324 & 2.08 \\
\hline hsa_circ_0085305 & 6.19 & hsa_circ_0050038 & 2.45 & hsa_circ_0062048 & 3.18 & hsa_circ_0054580 & 2.07 \\
\hline hsa_circ_0057390 & 5.90 & hsa_circ_0071886 & 2.44 & hsa_circ_0089436 & 3.16 & hsa_circ_0034935 & 2.07 \\
\hline hsa_circ_0000199 & 5.83 & hsa_circ_0062764 & 2.44 & hsa_circ_0057547 & 3.15 & hsa_circ_0041675 & 2.07 \\
\hline hsa_circ_0064530 & 5.78 & hsa_circ_0064577 & 2.44 & hsa_circ_0023087 & 3.12 & hsa_circ_0085920 & 2.07 \\
\hline hsa_circ_0087204 & 5.70 & hsa_circ_0010505 & 2.43 & hsa_circ_0074647 & 3.10 & hsa_circ_0066825 & 2.07 \\
\hline hsa_circ_0049854 & 5.46 & hsa_circ_0072744 & 2.42 & hsa_circ_0058206 & 3.09 & hsa_circ_0038009 & 2.07 \\
\hline hsa_circ_0066604 & 5.40 & hsa_circ_0020205 & 2.42 & hsa_circ_0030862 & 3.08 & hsa_circ_0025898 & 2.07 \\
\hline hsa_circ_0012896 & 5.26 & hsa_circ_0031925 & 2.42 & hsa_circ_0082066 & 3.08 & hsa_circ_0071382 & 2.07 \\
\hline hsa_circ_0088722 & 5.20 & hsa_circ_0076628 & 2.42 & hsa_circ_0013043 & 3.07 & hsa_circ_0071821 & 2.07 \\
\hline hsa_circ_0027791 & 5.07 & hsa_circ_0027007 & 2.41 & hsa_circ_0057445 & 3.06 & hsa_circ_0076610 & 2.07 \\
\hline hsa_circ_0021491 & 4.99 & hsa_circ_0074034 & 2.41 & hsa_circ_0026358 & 3.06 & hsa_circ_0058788 & 2.06 \\
\hline hsa_circ_0045275 & 4.92 & hsa_circ_0044837 & 2.40 & hsa_circ_0078639 & 3.05 & hsa_circ_0059268 & 2.06 \\
\hline hsa_circ_0054444 & 4.86 & hsa_circ_0023450 & 2.40 & hsa_circ_0013239 & 3.03 & hsa_circ_0032021 & 2.06 \\
\hline hsa_circ_0090097 & 4.75 & hsa_circ_0066676 & 2.40 & hsa_circ_0089437 & 3.02 & hsa_circ_0050636 & 2.06 \\
\hline hsa_circ_0069415 & 4.66 & hsa_circ_0071409 & 2.39 & hsa_circ_0026539 & 3.00 & hsa_circ_0090811 & 2.05 \\
\hline hsa_circ_0028116 & 4.63 & hsa_circ_0010800 & 2.39 & hsa_circ_0044566 & 2.98 & hsa_circ_0081975 & 2.05 \\
\hline hsa_circ_0019256 & 4.55 & hsa_circ_0041954 & 2.39 & hsa_circ_0086587 & 2.97 & hsa_circ_0082296 & 2.05 \\
\hline hsa_circ_0023258 & 4.44 & hsa_circ_0049461 & 2.37 & hsa_circ_0016072 & 2.96 & hsa_circ_0018529 & 2.05 \\
\hline hsa_circ_0074783 & 4.44 & hsa_circ_0002588 & 2.36 & hsa_circ_0021493 & 2.95 & hsa_circ_0075248 & 2.05 \\
\hline hsa_circ_0056946 & 4.44 & hsa_circ_0081148 & 2.36 & hsa_circ_0077048 & 2.94 & hsa_circ_0047176 & 2.05 \\
\hline hsa_circ_0028450 & 4.18 & hsa_circ_0022846 & 2.36 & hsa_circ_0013042 & 2.93 & hsa_circ_0000298 & 2.05 \\
\hline hsa_circ_0080225 & 4.09 & hsa_circ_0087813 & 2.36 & hsa_circ_0005446 & 2.91 & hsa_circ_0054586 & 2.05 \\
\hline hsa_circ_0043816 & 4.08 & hsa_circ_0054919 & 2.36 & hsa_circ_0075038 & 2.89 & hsa_circ_0040430 & 2.04 \\
\hline hsa_circ_0027793 & 3.99 & hsa_circ_0030867 & 2.35 & hsa_circ_0049379 & 2.87 & hsa_circ_0050434 & 2.04 \\
\hline hsa_circ_0054585 & 3.92 & hsa_circ_0044346 & 2.35 & hsa_circ_0023871 & 2.87 & hsa_circ_0026013 & 2.04 \\
\hline hsa_circ_0006919 & 3.87 & hsa_circ_0024745 & 2.35 & hsa_circ_0039224 & 2.87 & hsa_circ_0017079 & 2.04 \\
\hline hsa_circ_0002292 & 3.86 & hsa_circ_0046087 & 2.34 & hsa_circ_0031931 & 2.86 & hsa_circ_0006782 & 2.04 \\
\hline hsa_circ_0014848 & 3.79 & hsa_circ_0020073 & 2.33 & hsa_circ_0056286 & 2.86 & hsa_circ_0074306 & 2.03 \\
\hline hsa_circ_0091746 & 3.72 & hsa_circ_0033841 & 2.33 & hsa_circ_0051190 & 2.85 & hsa_circ_0026538 & 2.03 \\
\hline hsa_circ_0003756 & 3.70 & hsa_circ_0039053 & 2.32 & hsa_circ_0060837 & 2.85 & hsa_circ_0027021 & 2.03 \\
\hline hsa_circ_0039991 & 3.68 & hsa_circ_0047223 & 2.31 & hsa_circ_0085783 & 2.84 & hsa_circ_0058208 & 2.03 \\
\hline hsa_circ_0043112 & 3.68 & hsa_circ_0028512 & 2.31 & hsa_circ_0027127 & 2.82 & hsa_circ_0051506 & 2.03 \\
\hline hsa_circ_0074793 & 3.67 & hsa_circ_0076212 & 2.30 & hsa_circ_0086926 & 2.82 & hsa_circ_0090466 & 2.02 \\
\hline hsa_circ_0016097 & 3.67 & hsa_circ_0085965 & 2.29 & hsa_circ_0039135 & 2.81 & hsa_circ_0090810 & 2.02 \\
\hline hsa_circ_0089442 & 3.64 & hsa_circ_0006836 & 2.28 & hsa_circ_0007821 & 2.81 & hsa_circ_0041521 & 2.02 \\
\hline hsa_circ_0091740 & 3.63 & hsa_circ_0040008 & 2.26 & hsa_circ_0063090 & 2.79 & hsa_circ_0063349 & 2.02 \\
\hline hsa_circ_0054559 & 3.63 & hsa_circ_0022735 & 2.26 & hsa_circ_0045283 & 2.78 & hsa_circ_0038133 & 2.02 \\
\hline hsa_circ_0091395 & 3.62 & hsa_circ_0037191 & 2.26 & hsa_circ_0026544 & 2.77 & hsa_circ_0080498 & 2.01 \\
\hline hsa_circ_0003859 & 3.59 & hsa_circ_0030853 & 2.25 & hsa_circ_0029327 & 2.76 & hsa_circ_0066386 & 2.01 \\
\hline hsa_circ_0028501 & 3.57 & hsa_circ_0049004 & 2.25 & hsa_circ_0023307 & 2.75 & hsa_circ_0083607 & 2.01 \\
\hline hsa_circ_0045270 & 3.57 & hsa_circ_0040046 & 2.25 & hsa_circ_0048966 & 2.75 & hsa_circ_0058209 & 2.00 \\
\hline hsa_circ_0017784 & 3.54 & hsa_circ_0038718 & 2.25 & hsa_circ_0029337 & 2.75 & hsa_circ_0087806 & 2.00 \\
\hline hsa_circ_0018965 & 2.16 & hsa_circ_0082215 & 2.25 & hsa_circ_0058599 & 2.75 & hsa_circ_0072904 & 2.21 \\
\hline hsa_circ_0073166 & 2.16 & hsa_circ_0027307 & 2.24 & hsa_circ_0057418 & 2.74 & hsa_circ_0080982 & 2.21 \\
\hline hsa_circ_0085620 & 2.16 & hsa_circ_0058883 & 2.24 & hsa_circ_0019196 & 2.72 & hsa_circ_0036610 & 2.19 \\
\hline hsa_circ_0010096 & 2.16 & hsa_circ_0044462 & 2.24 & hsa_circ_0030860 & 2.70 & hsa_circ_0085922 & 2.19 \\
\hline hsa_circ_0083694 & 2.16 & hsa_circ_0071759 & 2.24 & hsa_circ_0003044 & 2.69 & hsa_circ_0050674 & 2.19 \\
\hline hsa_circ_0025983 & 2.16 & hsa_circ_0051784 & 2.24 & hsa_circ_0001225 & 2.68 & hsa_circ_0061143 & 2.19 \\
\hline hsa_circ_0079100 & 2.15 & hsa_circ_0017865 & 2.23 & hsa_circ_0067068 & 2.68 & hsa_circ_0040112 & 2.19 \\
\hline hsa_circ_0006559 & 2.15 & hsa_circ_0032326 & 2.23 & hsa_circ_0026408 & 2.67 & hsa_circ_0029322 & 2.18 \\
\hline hsa_circ_0008900 & 2.15 & hsa_circ_0018702 & 2.23 & hsa_circ_0024365 & 2.67 & hsa_circ_0051193 & 2.18 \\
\hline hsa_circ_0007778 & 2.15 & hsa_circ_0080497 & 2.22 & hsa_circ_0067085 & 2.66 & hsa_circ_0076797 & 2.18 \\
\hline hsa_circ_0065419 & 2.14 & hsa_circ_0005366 & 2.22 & hsa_circ_0037664 & 2.66 & hsa_circ_0008675 & 2.18 \\
\hline hsa_circ_0055400 & 2.14 & hsa_circ_0027033 & 2.22 & hsa_circ_0029348 & 2.65 & hsa_circ_0080834 & 2.17 \\
\hline hsa_circ_0036613 & 2.14 & hsa_circ_0046970 & 2.21 & hsa_circ_0023452 & 2.65 & hsa_circ_0057010 & 2.17 \\
\hline hsa_circ_0049342 & 2.14 & hsa_circ_0067573 & 2.21 & hsa_circ_0064826 & 2.65 & hsa_circ_0074307 & 2.17 \\
\hline hsa_circ_0051516 & 2.14 & hsa_circ_0063118 & 2.21 & hsa_circ_0089428 & 2.63 & hsa_circ_0087713 & 2.17 \\
\hline hsa_circ_0052131 & 2.14 & hsa_circ_0078356 & 2.13 & hsa_circ_0013333 & 2.63 & hsa_circ_0049390 & 2.58 \\
\hline hsa_circ_0084064 & 2.14 & hsa_circ_0046756 & 2.13 & hsa_circ_0017635 & 2.62 & hsa_circ_0074649 & 2.58 \\
\hline hsa_circ_0072128 & 2.13 & hsa_circ_0044524 & 2.12 & hsa_circ_0070517 & 2.61 & hsa_circ_0062146 & 2.57 \\
\hline hsa_circ_0002684 & 2.13 & hsa_circ_0076099 & 2.12 & hsa_circ_0088705 & 2.59 & hsa_circ_0031928 & 2.56 \\
\hline hsa_circ_0084126 & 2.12 & hsa_circ_0013063 & 2.12 & hsa_circ_0009925 & 2.59 & hsa_circ_0081827 & 2.56 \\
\hline
\end{tabular}

host genes [14]. Therefore, GO analysis and pathway analysis of the host genes of circRNAs could provide some insights into the function of circRNAs. In this study, we showed that the significant enriched GO term of host genes of dysregulated circRNAs in biological process was extracellular matrix organization (Fig. 2A). The most significant enriched GO term in cellular component was cytoplasm (Fig. 2B). The most significant enriched GO term in molecular function was protein binding (Fig. 2C). Pathway analysis revealed the top 10 signaling pathway potentially involved in dysregulated circRNAs-mediated regulatory network. Of them, focal adhesion signaling pathway was ranked the top 1 (Fig. 2D). 


\section{Cellular Physiology Cell Physiol Biochem 2018:47:1630-1642 \begin{tabular}{l|l} 
and Biochemistry Published online: June 27, 2018 & $\begin{array}{l}\text { C 2018 The Author(s). Published by S. Karger AG, Basel } \\
\text { www.karger.com/cpb }\end{array}$ \\
\hline
\end{tabular} \\ Li et al.: CircRNA and Pterygium}

miRNA response elements analysis of dysregulated expressed circRNAs in pterygium

Accumulating evidence has shown that several circRNAs contains conserved seed matches for miRNAs, and may function as microRNA (miRNA) sponges to play their biological roles [11]. We predicted whether dysregulated circRNAs may play their biological roles by acting as miRNA sponges in pterygium. circRNA/miRNA interaction was predicted based on TargetScan and miRana. We predicted whether the top 10 up-regulated circRNAs and the top 10 down-regulated circRNAs could bind to miRNAs. The results demonstrated that 16 circRNAs could potentially bind to at least five different miRNAs (Table 2), suggesting that most of dysregulated circRNAs could function as miRNA sponges to regulate the expression of target genes in pterygium.

\section{circ_0085020 is shown as a potential biomarker for pterygium}

The above-mentioned result showed that circ_0085020 was ranked among the top upregulated circRNA in pterygium samples. We then collected 50 paired pterygium and normal conjunctiva to further evaluate the clinical relevance of circ_0085020. All of the patients had the untreated primary pterygium. They have been accepted conventional excision of pterygium with auto-transplantation of conjunctiva. The patients included 22 female (Age: $41.2 \pm 6.6$ year) and 28 males (Age: $39.5 \pm 4.3$ year). The corneal ingrowth in patients was ranging from $2 \mathrm{~mm}$ to $4 \mathrm{~mm}$. The results showed that circ_0085020 expression was significantly up-regulated in pterygium compared with normal conjunctiva sample (Fig. 3).

\section{circ_0085020 regulates fibroblast function in pterygium}

Pterygium is an ocular surface condition characterized by abnormal fibroblast proliferation and matrix deposition [8]. We thus investigated the role of circ_0085020 in pterygium fibroblast function. circ_0085020 siRNA transfection significantly down-regulated circ_0085020 expression but did not affect the expression of host gene, LAPTM4B (Fig. 4A and 4B). MTT assays showed that circ_0085020 silencing significantly decreased pterygium fibroblast cell viability (Fig. 4C). Ki67 staining assays showed that circ_0085020 silencing significantly decreased pterygium fibroblast proliferation (Fig. 4D). Transwell assays revealed that circ_0085020 silencing inhibited pterygium fibroblast migration (Fig. 4E). Ultraviolet radiation exposure is an important risk factor for pterygium [24]. We also determined whether circ_0085020 regulated the development of UV-induced apoptosis using Hoechst

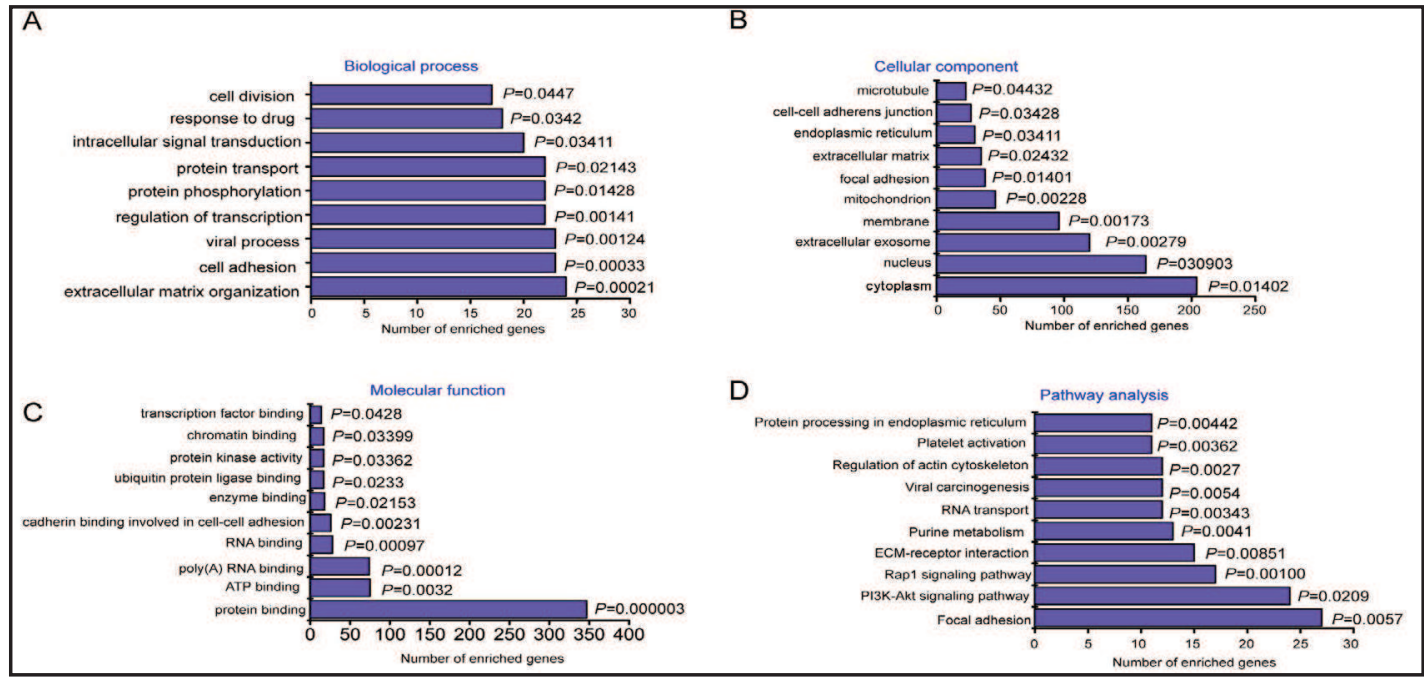

Fig. 2. GO enrichment and pathway analysis of the host genes of dysregulated circRNAs (A-C) GO enrichment analysis shows the vocabulary to describe differentially expressed circRNAs between paired conjunctiva and pterygium samples. The ontology covers three different domains: biological process (A), cellular component (B), and molecular function (C). (D) The bar plot displays the result of signaling pathway analysis, which demonstrates the top 10 signaling pathways involved in circRNA-mediated regulatory network in pterygium. 


\begin{tabular}{|c|c|c|}
\hline$c e$ & Cell Physiol Biochem 201 & 7:1630-1642 \\
\hline nd Biochemistrv & $\begin{array}{l}\text { DOI: 10.1159/000490982 } \\
\text { Published online: June 27, } 2018\end{array}$ & $\begin{array}{l}\text { O } 2018 \text { The Author(s). Published by S. Karger AG, Basel } \\
\text { www.karger.com/cpb }\end{array}$ \\
\hline
\end{tabular}

Table 2. Identification of potential miRNAs which can bind to dysregulated circRNAs

\begin{tabular}{|c|c|c|c|c|c|c|c|}
\hline circRNA & Gene symbol & Fold Change & MRE1 & MRE2 & MRE3 & MRE4 & MRE5 \\
\hline \multicolumn{8}{|l|}{ Up-regulated } \\
\hline hsa_circ_0085019 & LAPTM4B & 17.01 & miR-625-5p & $\operatorname{miR}-1275$ & miR-1910-3p & miR-6511a-5p & miR-4665-5p \\
\hline hsa_circ_0010170 & MFAP2 & 12.01 & miR-6893-5p & miR-6808-5p & miR-940 & miR-873-5p.2 & miR-1291 \\
\hline hsa_circ_0087653 & CTSL2 & 10.71 & miR-296-5p & miR-3918 & miR-6772-3p & miR-4448 & miR-4786-3p \\
\hline hsa_circ_0085013 & LAPTM4B & 8.90 & miR-6816-3p & miR-3127-5p & miR-4695-5p & miR-4266 & miR-5187-5p \\
\hline hsa_circ_0085020 & LAPTM4B & 8.88 & miR-625-5p & miR-1275 & miR-1910-3p & miR-6511a-5p & miR-4665-5p \\
\hline hsa_circ_0031978 & CDKN3 & 8.05 & miR-4659a-3p & miR-4659b-3p & $\operatorname{miR}-548 \mathrm{q}$ & miR-4520-2-3p & miR-4519 \\
\hline hsa_circ_0069798 & NMU & 7.98 & miR-4778-3p & miR-4480 & miR-5000-3p & miR-4436a & miR-663b \\
\hline hsa_circ_0015295 & GAS5 & 7.05 & miR-3620-3p & miR-6851-3p & miR-597-3p & miR-1205 & miR-7160-5p \\
\hline hsa_circ_0015288 & GAS5 & 7.03 & miR-766-3p & miR-3925-3p & miR-515-5p & miR-519e-5p & miR-6875-3p \\
\hline hsa_circ_0015310 & GAS5 & 6.90 & miR-6810-5p & miR-6870-3p & miR-3117-3p & miR-18a-5p & miR-18b-5p \\
\hline \multicolumn{8}{|l|}{ Down-regulated } \\
\hline hsa_circ_0087198 & GDA & 79.74 & - & - & - & - & - \\
\hline hsa_circ_0025454 & CD69 & 26.11 & miR-6881-3p & miR-7111-3p & miR-6780a-3p & miR-3130-5p & miR-4482-5p \\
\hline hsa_circ_0002141 & ZDBF2 & 12.13 & miR-2861 & miR-3654 & miR-124-3p.1 & miR-506-3p & miR-124-3p.2 \\
\hline hsa_circ_0056159 & IL1RN & 11.10 & - & - & - & - & - \\
\hline hsa_circ_0064840 & ITGA9 & 9.40 & miR-4675 & $\operatorname{miR}-4741$ & miR-376a-3p & miR-376b-3p & miR-6875-3p \\
\hline hsa_circ_0034472 & THBS1 & 9.38 & miR-4736 & miR-3064-3p & miR-6869-3p & miR-3975 & miR-1908-3p \\
\hline hsa_circ_0009558 & ERRFI1 & 9.08 & miR-93-3p & miR-6071 & miR-6515-3p & miR-3912-5p & miR-5693 \\
\hline hsa_circ_0062162 & CECR2 & 8.87 & miR-7111-3p & miR-877-3p & miR-6881-3p & miR-6875-3p & miR-1231 \\
\hline hsa_circ_0088344 & sтом & 8.20 & - & - & - & - & - \\
\hline hsa_circ_0087198 & C9orf150 & 79.74 & - & - & - & - & - \\
\hline
\end{tabular}

33342 and Calcein-AM/PI staining. circ_0085020 silencing further increased UV-induced pterygium fibroblast cell apoptosis as shown by increased apoptotic nuclei (condensed or fragmented) and PI-positive cells (dying or dead cells) (Fig. 4F and 4G).

\section{circ_0085020 regulates epithelial function in pterygium}

The epithelial cells also play an important role in the progression of pterygium, which is associated with the invasive and recurrent behavior [2]. circ_0085020 siRNA transfection down-regulated circ_0085020 expression but did not affect the expression of the host gene, LAPTM4B, in pterygium epithelial cells (Fig. 5A and 5B). circ_0085020 silencing significantly decreased pterygium epithelial viability (Fig. 5C), decreased pterygium epithelial proliferation (Fig. 5D), and inhibited pterygium epithelial migration (Fig. 5E). Moreover, circ_0085020 was involved in

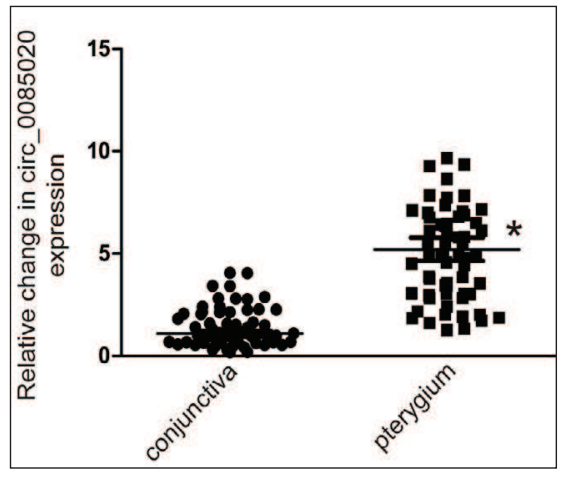

Fig. 3. circ_0085020 is shown as a potential biomarker for pterygium qRT-PCR assays were conducted to compare the expression difference of circ_0085020 between 50 paired pterygium and 50 normal conjunctiva $\left({ }^{*} P<0.05\right.$ versus conjunctiva). the regulation of epithelial apoptosis. circ_0085020 silencing further increased UV-induced pterygium epithelial apoptosis as shown by increased apoptotic nuclei (condensed or fragmented) and PI-positive cells (dying or dead cells) (Fig. $5 \mathrm{~F}$ and $5 \mathrm{G})$.

\section{Discussion}

Pterygium has very complicated pathogenesis, including extracellular matrix remodeling, inflammation, angiogenesis, aberrant proliferation, and apoptosis $[1,8]$. However, the precise mechanism of pterygium formation and development is not fully understood. In this study, we determined circRNA expression profiling between paired conjunctiva and pterygium. Approximately 669 circRNAs were found to be abnormally expressed in pterygium. circ_0085020 (circ-LAPTM4B) was further verified to significantly be up-regulated in pterygium. circ_0085020 (circ-LAPTM4B) was involved in the pathogenesis of pterygium through regulating pterygium fibroblast and epithelial cell function.

circRNAs are usually generated from the exonic or intronic sequences. They can play their roles by acting as regulators of parental gene expression, regulators of splicing and transcription, or miRNA sponges [11]. Thus, it is possible to predict the functions of serveral 


\section{Cellular Physiology Cell Physiol Biochem 2018;47:1630-1642 \begin{tabular}{l|l|l} 
and Biochemistry & Dublished online: June 27, 2018 & $\begin{array}{l}\text { (0) } 2018 \text { The Author(s). Published by S. Karger AG, Basel } \\
\text { www.karger.com/cpb }\end{array}$ \\
\cline { 2 - 3 }
\end{tabular}}

Li et al.: CircRNA and Pterygium

circRNAs via their parental genes. GO enrichment analysis showed that the most enriched GO term in biological process was extracellular matrix organization. The most enriched GO term in cellular component was cytoplasm. The most enriched GO term in molecular function was protein binding. Extracellular matrix remodeling via the actions of matrix metalloproteinases has been implicated in the pathogenesis of pterygia $[8,25]$. After biogenesis, circRNAs can be efficiently transported to the cytosol since the endogenous circRNAs are usually localized

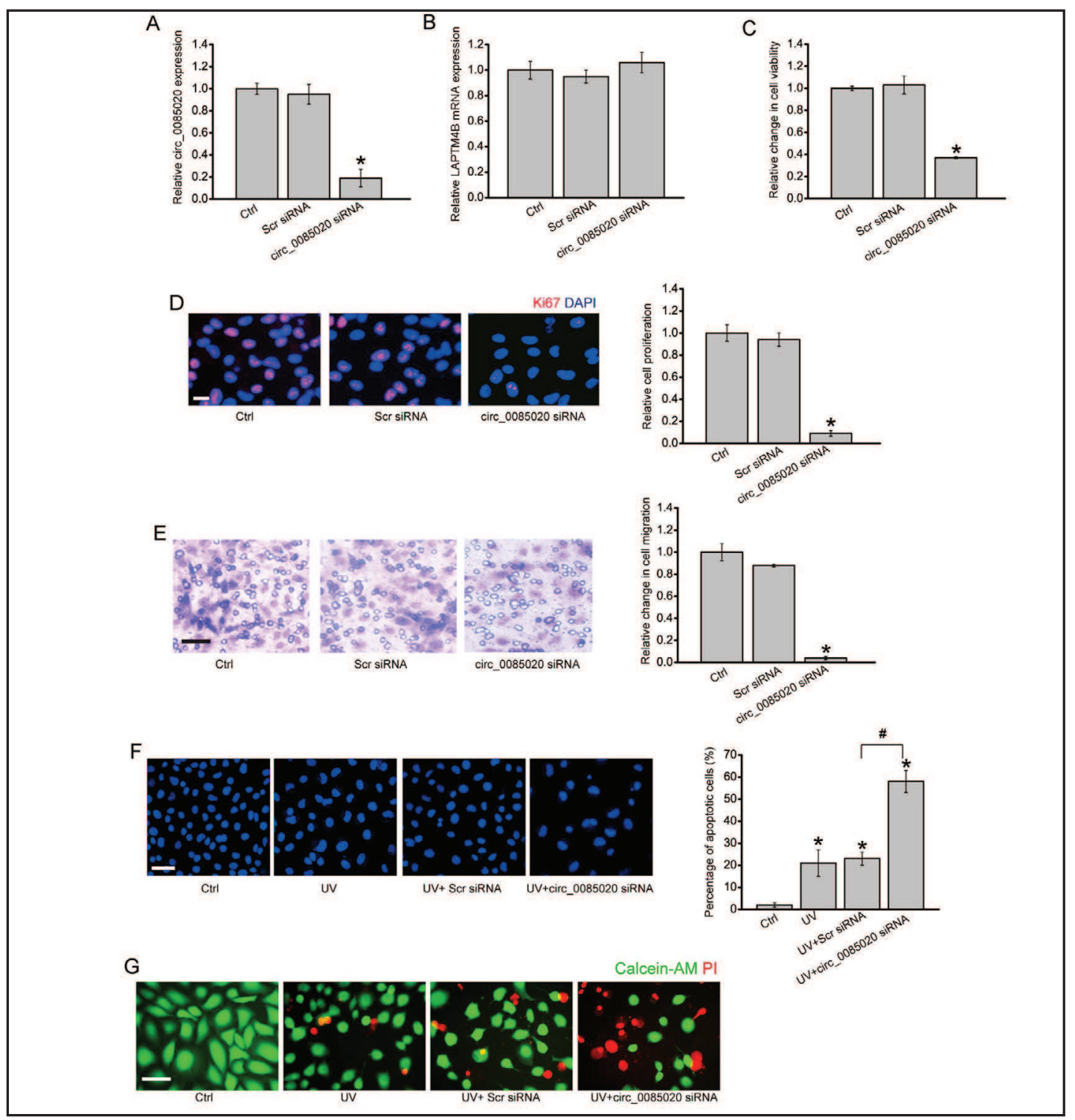

Fig. 4. circ_0085020 regulates fibroblast function in pterygium(A and B) Pterygium fibroblasts were transfected with scrambled (Scr) siRNA, siRNA targeting the backsplice sequence of circ_0085020, or left untreated (Ctrl) for $24 \mathrm{~h}$. qRT-PCRs were conducted to detect circ_0085020 (A, n=4, ${ }^{*} P<0.05$ versus Ctrl group) and LAPTM4B mRNA expression (B, $\mathrm{n}=4,{ }^{*} P 05$ versus Ctrl group). (C) MTT assays were conducted to detect cell viability ( $\mathrm{n}=4,{ }^{*} P<0.05$ versus Ctrl group). (D) Cell proliferation was detected by Ki67 staining ( $\mathrm{n}=4$, ${ }^{*} P<0.05$ versus $C$ trl group). Scale bar, $10 \mu \mathrm{m}$. (E) Transwell assay and quantification analysis was conducted to detect cell migration ( $\mathrm{n}=4,{ }^{*} P<0.05$ versus Ctrl group). Scale bar, $50 \mu \mathrm{m}$. (F) Apoptotic cells were analyzed using Hoechst staining. The data was shown as means \pm SEM, and represented four individual experiments in which >300 cells were counted. Scale bar: $20 \mu \mathrm{m}$. (G) Apoptotic cells were detected by PI/Calcein-AM double staining. Scale bar: $50 \mu \mathrm{m}$. The significant difference was evaluated by one-way ANOVA followed by post-hoc Bonferroni's test comparison test. \# indicated significance differences between the marked group. 


\section{Cellular Physiology Cell Physiol Biochem 2018;47:1630-1642

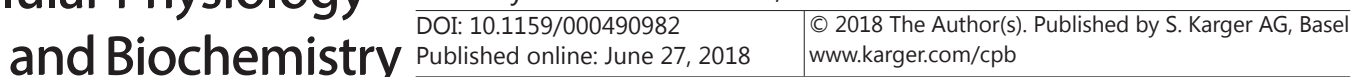

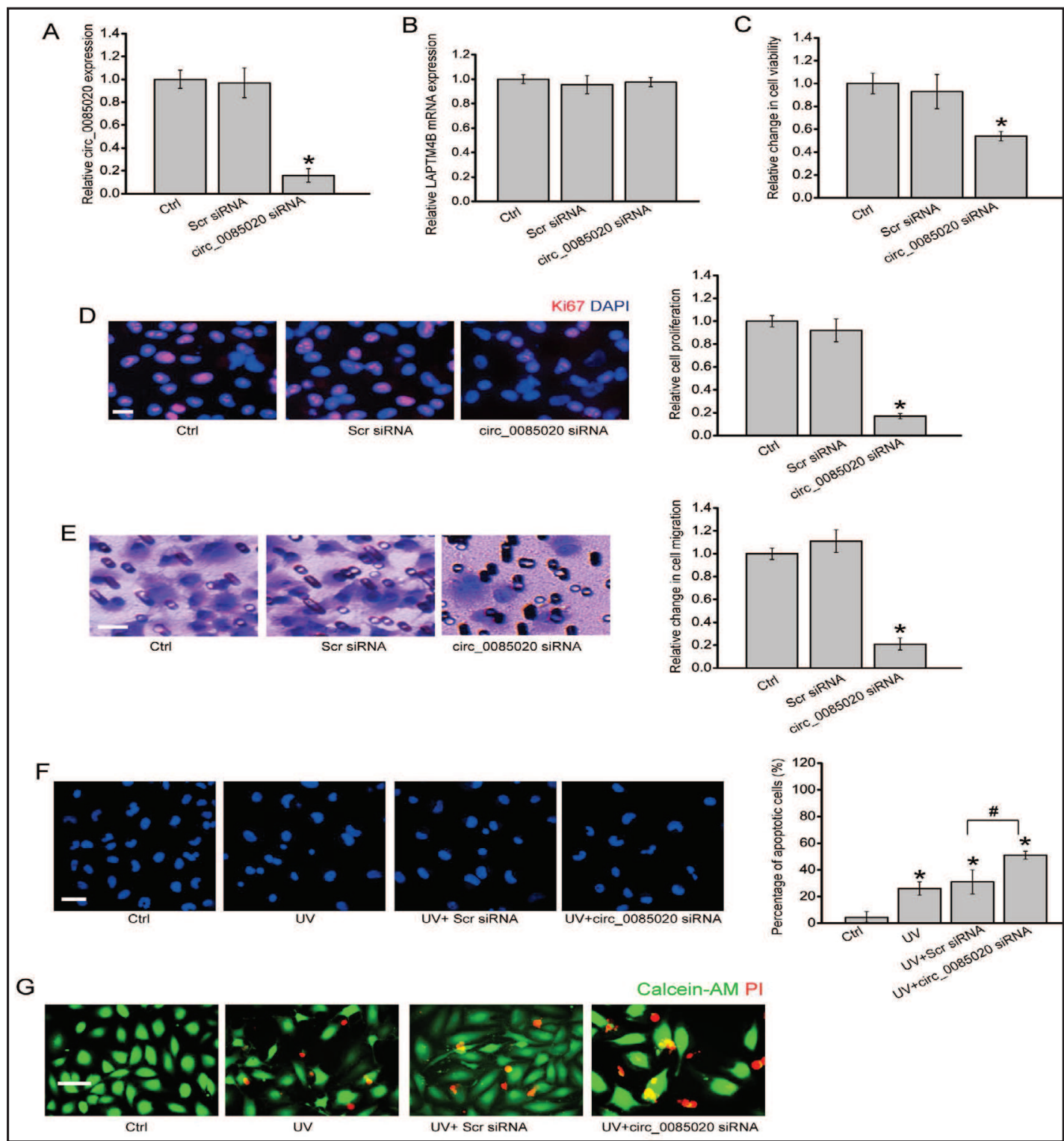

Fig. 5. circ_0085020 regulates epithelial cell function in pterygium(A and B) Pterygium epithelium was transfected with scrambled (Scr) siRNA, siRNA targeting the backsplice sequence of circ_0085020, or left untreated (Ctrl) for $24 \mathrm{~h}$. qRT-PCRs were conducted to detect circ_0085020 (A, n=4, ${ }^{*} P<0.05$ versus Ctrl group) and LAPTM4B mRNA expression (B, $n=4,{ }^{*} P<0.05$ versus $C$ trl group). (C) MTT assays were conducted to detect cell viability ( $\mathrm{n}=4,{ }^{*} P<0.05$ versus $\mathrm{Ctrl}$ group). (D) Cell proliferation was detected by Ki67 staining ( $\mathrm{n}=4,{ }^{*} P<0.05$ versus $\mathrm{Ctrl}$ group). Scale bar, $10 \mu \mathrm{m}$. (E) Transwell assay and quantification analysis was conducted to detect cell migration ( $\mathrm{n}=4,{ }^{*} P<0.05$ versus Ctrl group). Scale bar, $50 \mu \mathrm{m}$. (F) Apoptotic cells were analyzed using Hoechst staining. The data was shown as means \pm SEM, and represented four individual experiments in which $>300$ cells were counted. Scale bar: $20 \mu \mathrm{m}$. (G) Apoptotic cells were detected by PI/ Calcein-AM double staining. Scale bar: $50 \mu \mathrm{m}$. The significant difference was evaluated by one-way ANOVA followed by post-hoc Bonferroni's test comparison test. \# indicated significance differences between the marked group.

to cytoplasm $[26,27]$ circRNA-mediated regulatory network would occur in cytoplasm, and affect the formation of pterygia via regulating extracellular matrix remodeling. Pathway analysis showed that focal adhesion signaling pathway was ranked the top 1 signaling pathway affected by circRNA-mediated network. Focal adhesion kinase (FAK) is a non- 


\section{Cellular Physiology Cell Physiol Biochem 2018:47:1630-1642 \begin{tabular}{c|c} 
DOI 10.1159/000490982 & a 2018 The Author(s) \\
and Biocherger.com/cpb
\end{tabular}

receptor tyrosine kinase, which is known for its role in adhesion-mediated signaling. It plays important roles in matrix remodeling, and potentially regulates fibroblast cell and epithelial cell function $[28,29]$. Based on the above-mentioned evidence, we speculated that circRNAmediated regulatory network is involved in the pathogenesis of pterygium.

The majority of circRNAs possesses miRNA response elements (MREs), and have miRNA-binding potentials, which increases the complexity of RNA regulatory network [30]. Previous studies have shown that CDR1as harbors more than 70 conventional miR7 binding sites [31]. circ-ITCH functions as miRNA (miR-7, miR-17, and miR-214) sponges and plays an inhibitory role in esophageal squamous cell carcinoma [32]. circHIPK3 is observed to sponge to 9 miRNAs with 18 potential binding sites [33]. We show that most of differentially expressed circRNAs in pterygium contain more than five different miRNA binding sites. miRNAs play important role in many cellular processes including cell growth, proliferation, differentiation, death, and apoptosis [34]. The role of miRNAs in pterygium has also been widely accepted $[6,35,36]$. circRNAs could compete with endogenous miRNAs by direct binding, resulting in increased levels of miRNA targets. Thus, it is not surprising that circRNA-miRNA-mRNA network is involved in the pathogenesis of pterygium.

circRNAs are specifically expressed in a developmental stage- and tissue-specific manner [37]. Unlike linear RNAs, circRNAs are very stable and resistant to RNase R. They are more suitable for serving as diagnostic or predictive biomarkers of human diseases [38]. Emerging evidence shows that circRNAs exhibit aberrant expression in atherosclerotic vascular disease, neurological disorders, and cancers, and serve as diagnostic or predictive biomarkers of these diseases $[39,40]$. circRNAs can be detected in plasma, saliva, and blood [41-43]. Abnormal circRNA expression in disease condition may reflect either a conditionspecific transcriptome change or a direct consequence of circRNA release from the diseased tissue. circ_0085020 (circ-LAPTM4B) expression is significantly up-regulated in pterygium compared with paired conjunctiva. We speculated that circ_0085020 (circ-LAPTM4B) silencing may be beneficial for the therapeutic intervention of pterygium.

Pterygium is usually characterized by abnormal fibroblast proliferation and matrix deposition. Its clinical severity is tightly associated with the fleshiness or amount of fibrous tissue [35]. Previous studies have shown that pterygium fibroblasts display characteristics of transformed cells, including loss of heterozygosity and microsatellite instability [44]. circ_0085020 (circ-LAPTM4B) up-regulation could significantly increase pterygium fibroblast cell proliferation and migration ability. Thus, we speculated that compared to normal conjunctival epithelial cells, circ_0085020 (circ-LAPTM4B) up-regulation epithelium would take longer to reach senescence. Fibroblast proliferation in pterygium would lead to increased matrix production, resulting in increased fleshy lesion. The epithelial cells play a crucial role in the formation of pterygium, which are responsible for the invasive and recurrent behavior of pterygium via regulating the secretion of matrix metalloproteinases (MMPs) and tissue inhibitors of MMPs (TIMPs) [8]. Moreover, abnormal apoptosis is shown in pterygium compared with the normal conjunctiva. Chronic UV exposure plays a major role in the pathogenesis of pterygium [45]. circ_0085020 (circ-LAPTM4B) up-regulation could prevent pterygium epithelium against UV-induced apoptosis. Previous study has revealed the presence of an anti-apoptotic protein Bcl-2 in pterygium epithelium but not conjunctiva, which counteracts the pro-apoptotic protein Bax [46, 47]. We speculated that circ_0085020 (circ-LAPTM4B) might play an anti-apoptotic role in the pathogenesis of pterygium similar as Bcl-2.

In conclusion, this study provides important evidence that circular RNAs are involved in the pathogenesis of pterygium. circ_0085020 (circ-LAPTM4B) up-regulation leads to abnormal pterygium fibroblast and epithelial function. Our finding may be applied for the treatment of pterygium. It may be possible to arrest pterygium formation by non-surgical treatment using the inhibitor of circ_0085020 (circ-LAPTM4B). In the fleshy pterygium lesions, these strategies may even play a role in the prevention of post-surgical recurrence after excision. 


\section{Cellular Physiology Cell Physiol Biochem 2018:47:1630-1642 \begin{tabular}{l|l} 
and Biochemistry Published online: June 27, 2018 & $\begin{array}{l}\text { ( 2)18 The Author(s). Published by S. Karger AG, Basel } \\
\text { www.karger.com/cpb }\end{array}$ \\
\hline
\end{tabular} \\ Li et al.: CircRNA and Pterygium}

\section{Acknowledgements}

This work was generously supported by the grants from the National Natural Science Foundation of China (Grant No. 81371055 and 81570859 to Q.J., and Grant No. 81670878 to J.Y.) and Grants from innovation team Project Fund of Jiangsu Province (No. CXTDB2017010 to Q.J).

\section{Disclosure Statement}

The authors declare that they have no conflict of interests.

\section{References}

1 Chui J, Di Girolamo N, Wakefield D, Coroneo MT: The pathogenesis of pterygium: current concepts and their therapeutic implications. Ocul Surf 2008;6:24-43.

$\longrightarrow 2$ Liu T, Liu Y, Xie L, He X, Bai J: Progress in the pathogenesis of pterygium. Curr Eye Res 2013;38:1191-1197.

3 Hirst LW: The treatment of pterygium. Surv Ophthalmol 2003;48:145-180.

4 Gris O, Güell JL, del Campo Z: Limbal-conjunctival autograft transplantation for the treatment of recurrent pterygium. Ophthalmology 2000;107:270-273.

5 Hacıoğlu D, Erdöl H: Developments and current approaches in the treatment of pterygium. Int Ophthalmol 2017;37:1073-1081.

6 Lan W, Chen S, Tong L: MicroRNA-215 regulates fibroblast function: insights from a human fibrotic disease. Cell Cycle 2015; 14:1973-1984.

7 Ebbesen KK, Kjems J, Hansen TB: Circular RNAs: identification, biogenesis and function. Biochim Biophys Acta 2016;1859:163-168.

-8 Di Girolamo N, Chui J, Coroneo MT, Wakefield D: Pathogenesis of pterygia: role of cytokines, growth factors, and matrix metalloproteinases. Prog Retin Eye Res 2004;23:195-228.

-9 Yazar S, Cuellar-Partida G, McKnight CM, Quach-Thanissorn P, Mountain JA, Coroneo MT, Pennell CE, Hewitt AW, MacGregor S, Mackey DA: Genetic and environmental factors in conjunctival UV autofluorescence. JAMA Ophthalmol 2015;133:406-412.

10 Vicens Q, Westhof E: Biogenesis of circular RNAs. Cell 2014;159:13-14.

11 Salzman J: Circular RNA expression: its potential regulation and function. Trends Genet 2016;32:309-316.

12 Rybak-Wolf A, Stottmeister C, Glažar P, Jens M, Pino N, Giusti S, Hanan M, Behm M, Bartok O, Ashwal-Fluss R, Herzog M, Schreyer L, Papavasileiou P, Ivanov A, Öhman M, Refojo D, Kadener S, Rajewsky N: Circular RNAs in the mammalian brain are highly abundant, conserved, and dynamically expressed. Mol Cell 2015;58:870-885.

-13 Boeckel JN, Jaé N, Heumüller AW, Chen W, Boon RA, Stellos K, Zeiher AM, John D, Uchida S, Dimmeler S: Identification and characterization of hypoxia-regulated endothelial circular RNA. Circ Res 2015; 117:884890.

14 Barrett SP, Wang PL, Salzman J: Circular RNA biogenesis can proceed through an exon-containing lariat precursor. Elife 2015; 4:e07540.

15 Li H, Hao X, Wang H, Liu Z, He Y, Pu M, Zhang H, Yu H, Duan J, Qu S: Circular RNA expression profile of pancreatic ductal adenocarcinoma revealed by microarray. Cell Physiol Biochem 2016; 40:1334-1344.

-16 Fu L, Yao T, Chen Q Mo X, Hu Y, Guo J: Screening differential circular RNA expression profiles reveals hsa_ circ_0004018 is associated with hepatocellular carcinoma. Oncotarget 2017;8:58405-58416.

17 Lukiw WJ: Circular RNA (circRNA) in Alzheimer's disease (AD). Front Genet 2013;4:307.

18 Di Girolamo N, Tedla N, Kumar RK, McCluskey P, Lloyd A, Coroneo MT, Wakefield D: Culture and characterisation of epithelial cells from human pterygia. Br J Ophthalmol 1999;83:1077-1082.

-19 Solomon A, Grueterich M, Li DQ Meller D, Lee SB, Tseng SC: Overexpression of insulin-like growth factorbinding protein-2 in pterygium body fibroblasts. Invest OphthalmolVis Sci 2003;44:573-580.

20 Shan K, Jiang Q Wang XQ Wang YN, Yang H, Yao MD, Liu C, Li XM, Yao J, Liu B, Zhang YY, J Y, Yan B: Role of long non-coding RNA-RNCR3 in atherosclerosis-related vascular dysfunction. Cell Death Dis 2016;7:e2248.

-21 Yan B, Yao J, Liu JY, Li XM, Wang XQ, Li YJ, Tao ZF, Song YC, Chen Q, Jiang Q: LncRNA-MIAT regulates microvascular dysfunction by functioning as a competing endogenous RNA. Circ Res 2015;116:1143-1156. 


\section{Cellular Physiology Cell Physiol Biochem 2018:47:1630-1642

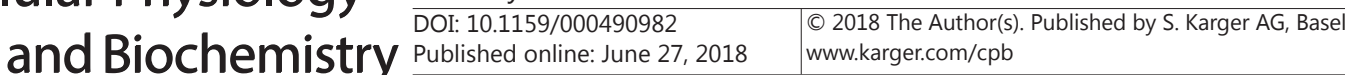 \\ Li et al.: CircRNA and Pterygium}

22 Yao J, Wang XQ Li YJ, Shan K, Yang H, Wang YN, Yao MD, Liu C, Li XM, Shen Y, Liu JY, Cheng H, Yuan J, Zhang YY, Jiang Q Yan B: Long non-coding RNA MALAT1 regulates retinal neurodegeneration through CREB signaling. EMBO Mol Med 2016;8:346-362.

23 Wang YN, Shan K, Yao MD, Yao J, Wang JJ, Li X, Liu B, Zhang YY, Ji Y, Jiang Q Yan B: Long noncoding RNAGAS5: a novel regulator of hypertension-induced vascular remodeling. Hypertension 2016;68:736-748.

24 Kim KW, Park SH, Kim JC: Fibroblast biology in pterygia. Exp Eye Res 2016;142:32-39.

25 Riau AK, Wong TT, Lan W, Finger SN, Chaurasia SS, Hou AH, Chen S, Yu SJ, Tong L: Aberrant DNA methylation of matrix remodeling and cell adhesion related genes in pterygium. PLoS One 2011;6:e14687.

-26 Hansen TB, Jensen TI, Clausen BH, Bramsen JB, Finsen B, Damgaard CK, Kjems J: Natural RNA circles function as efficient microRNA sponges. Nature 2013;495:384-388.

27 Hansen TB, Wiklund ED, Bramsen JB, Villadsen SB, Statham AL, Clark SJ, Kjems J: MiRNA-dependent gene silencing involving Ago2-mediated cleavage of a circular antisense RNA. EMBO J 2011;30:4414-4422.

-28 Larsen M, Artym VV, Green JA, Yamada KM: The matrix reorganized: extracellular matrix remodeling and integrin signaling. Curr Opin Cell Biol 2006;18:463-471.

29 Thiery JP, Sleeman JP: Complex networks orchestrate epithelial-mesenchymal transitions. Nat Rev Mol Cell Biol 2006;7:131-142.

30 Tay Y, Rinn J, Pandolfi PP: The multilayered complexity of ceRNA crosstalk and competition. Nature 2014;505:344-352.

-31 Xu H, Guo S, Li W, Yu P: The circular RNA Cdr1as, via miR-7 and its targets, regulates insulin transcription and secretion in islet cells. Sci Rep 2015;5:12453.

32 Li F, Zhang L, Li W, Deng J, Zheng J, An M, Lu J, Zhou Y: Circular RNA ITCH has inhibitory effect on ESCC by suppressing the Wnt/ $\beta$-catenin pathway. Oncotarget 2015;6:6001-6013.

-33 Zheng Q, Bao C, Guo W, Li S, Chen J, Chen B, Luo Y, Lyu D, Li Y, Shi G, Liang L, Gu J, He X, Huang S: Circular RNA profiling reveals an abundant circHIPK3 that regulates cell growth by sponging multiple miRNAs. Nat Commun 2016;7:11215.

-34 Winter J, Jung S, Keller S, Gregory RI, Diederichs S: Many roads to maturity: microRNA biogenesis pathways and their regulation. Nat Cell Biol 2009;11:228-234.

- 35 Chien KH, Chen SJ, Liu JH, Woung LC, Chen JT, Liang CM, Chiou SH, Tsai CY, Cheng CK, Hu CC, Peng CH: Correlation of microRNA-145 levels and clinical severity of pterygia. Ocul Surf 2013;11:133-138.

- 36 Engelsvold DH, Utheim TP, Olstad OK, Gonzalez P, Eidet JR, Lyberg T, Trøseid AM, Dartt DA, Raeder S: miRNA and mRNA expression profiling identifies members of the miR-200 family as potential regulators of epithelial-mesenchymal transition in pterygium. Exp Eye Res 2013;115:189-198.

-37 Chen LL: The biogenesis and emerging roles of circular RNAs. Nat Rev Mol Cell Biol 2016;17:205-211.

-38 Qu S, Yang X, Li X, Wang J, Gao Y, Shang R, Sun W, Dou K, Li H: Circular RNA: A new star of noncoding RNAs. Cancer Lett 2015;365:141-148.

-39 Lasda E, Parker R: Circular RNAs: diversity of form and function. RNA2014;20:1829-1842.

40 Zhang SJ, Chen X, Li CP, Li XM, Liu C, Liu BH, Shan K, Jiang Q Zhao C, Yan B: Identification and characterization of circular RNAs as a new class of putative biomarkers in diabetes retinopathy. Invest Ophthalmol Vis Sci 2017;58:6500-6509.

-41 Memczak S, Papavasileiou P, Peters 0, Rajewsky N: Identification and characterization of circular RNAs as a new class of putative biomarkers in human blood. PloS One 2015;10:e141214.

-42 Koh W, Pan W, Gawad C, Fan HC, Kerchner GA, Wyss-Coray T, Blumenfeld YJ, El-Sayed YY, Quake SR: Noninvasive in vivo monitoring of tissue-specific global gene expression in humans. Proc Natl Acad Sci USA 2014;111:7361-7366.

43 Bahn JH, Zhang Q Li F, Chan TM, Lin X, Kim Y, Wong DT, Xiao X: The landscape of microRNA, Piwiinteracting RNA, and circular RNA in human saliva. Clin Chem 2015;61:221-230.

-44 Spandidos DA, Sourvinos G, Kiaris H, Tsamparlakis J: Microsatellite instability and loss of heterozygosity in human pterygia. Br J Ophthalmol 1997;81:493-496.

45 Kwok LS, Coroneo MT: A model for pterygium formation. Cornea 1994;13:219-224.

46 Tan DT, Tang WY, Liu YP, Goh HS, Smith DR: Apoptosis and apoptosis related gene expression in normal conjunctiva and pterygium. Br J Ophthalmol 2000;84:212-216.

47 Liang K, Jiang Z, Ding BQ Cheng P, Huang DK, Tao LM: Expression of cell proliferation and apoptosis biomarkers in pterygia and normal conjunctiva. Mol Vis 2011;17:1687-1693. 